\title{
La estabilidad de los nuevos contratos indefinidos durante la crisis económica
}

\author{
INMACULADA CEBRIÁN LÓPEZ \\ Departamento de Fundamentos de Economía e Historia Económica, UNIVERSIDAD DE \\ ALCALÁ, ESPAÑA. E-mail: inmaculada.cebrian@uah.es
}

GLORIA MORENO RAYMUNDO

Departamento de Fundamentos de Economía e Historia Económica, UNIVERSIDAD DE ALCALÁ, ESPAÑA. E-mail: gloria.moreno@uah.es

\section{RESUMEN}

Este trabajo se centra en averiguar en qué medida la llegada de la crisis ha afectado al empleo estable que se inició en nuestro país en los años de la expansión económica en los que la tasa de crecimiento de la contratación indefinida fue mayor. El objetivo es detectar si se observan diferencias entre los distintos tipos de contratación indefinida que llevan asociados distintos costes de entrada y salida del empleo según la causa de baja. La base de datos utilizada procede del enlace de la Muestra Continua de Vidas Laborales con los registros de contratos del Servicio Público de Empleo Estatal. Los resultados muestran un volumen importante de inestabilidad asociado principalmente a puestos de trabajo de baja productividad en los que la salida es por despido.

Palabras clave: Contratación indefinida, duración.

\section{The Stability of the New Open Ended Contracts During the Economic Crisis}

\begin{abstract}
This paper is focused on how the economic crisis has concerned the stable employment that began in our country during the last years of economic expansion, when the rate of growth of the open ended contracts was the highest. The objective is to assess if it can be observed differences between the types of those open ended contracts with different costs of entry and exit to employment as well as to measure the incidence on their duration depending on special causes of exits. The database used is the "Continuous Sample of Working life" linked with registered contracts in Spanish Public Employment Service. The results show an important volume of instability associated mainly with jobs of low productivity and dismissals.
\end{abstract}

Keywords: Open Ended Contracts, Duration.

Clasificación JEL: J08, J18

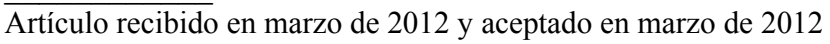

Artículo disponible en versión electrónica en la página www.revista-eea.net, ref. ə-30113 


\section{INTRODUCCIÓN}

Una de las características del mercado de trabajo español es que se encuentra segmentado entre trabajadores con una relación laboral indefinida y otros cuya relación es temporal. A pesar de que la crisis económica ha afectado en mayor medida a los trabajadores temporales, los indefinidos también se han visto considerablemente perjudicados, no sólo porque se han iniciado menos contratos sino porque una proporción de los que había han terminado.

La finalización de los contratos indefinidos puede llevar consigo un coste económico que puede tener diversas implicaciones sobre el mercado de trabajo. En primer lugar, existe un efecto sobre el empleo y, más concretamente, sobre la dinámica de entrada y salida en el empleo. De manera que cuanto mayores sean los costes del despido, menos transiciones entre ambas situaciones cabe esperar que haya. En España, la legislación sobre despidos establece condiciones diferentes para los trabajadores temporales y para los indefinidos, lo que en parte explica la segmentación del mercado laboral en estos dos colectivos con diferentes costes de ajuste.

Los costes de ajuste del factor trabajo y sus efectos sobre su grado de utilización en las empresas han sido un tema de permanente debate entre los estudiosos del mercado de trabajo, los políticos y los agentes sociales. A lo largo de las décadas de los ochenta y noventa el debate se centró en la búsqueda de una explicación a la elevada tasa de paro, su duración y su persistencia. Prácticamente todos incidían sobre el importante papel que tienen tanto los costes de contratación, como los costes de salida del puesto de trabajo, considerados ambos como factores determinantes de los movimientos que se producen en el mercado laboral. Aunque la teoría sugiere que los resultados finales son ambiguos, la tesis general ${ }^{1}$ parte de la idea de que la legislación sobre protección del empleo (costes de despido y de contratación) condiciona la eficacia del mercado de trabajo al introducir rigideces directas en el sistema que impiden que se resuelva el problema del desempleo, especialmente en el largo plazo. Y esto es así porque resulta indiscutible que las empresas que forman la demanda del factor trabajo, a la hora de contratar, consideran el efecto que estos costes pueden tener sobre sus beneficios futuros (Toharia, 1986).

Cuando una empresa termina su relación con un trabajador se producen diversos efectos negativos, tanto para el trabajador, como para la propia empresa. Por un lado, el efecto inmediato es que el trabajador deja de percibir su salario, por lo que su nivel de renta se ve reducido. A esto se añade que según cuál sea la duración del periodo que se mantenga desempleado tras esta salida, sufrirá una pérdida de capital humano que tendrá consecuencias para su empleo e in-

\footnotetext{
${ }^{1}$ Un resumen sobre el tipo de planteamientos elaborados en torno a estos temas, comparando Estados Unidos y Europa, se puede encontrar en los trabajos de Nickell (1997) o Siebert (1997).
} 
gresos futuros. Por otra parte, desde el punto de vista de la empresa, se generan costes relacionados con la rotación y la pérdida de la inversión que se haya podido realizar en capital específico, así como el coste que implica el pago de cualquier tipo de indemnización relacionada con la ruptura. Además, desde el punto de vista de la sociedad, existen también costes derivados de la salida de un trabajador de su empleo, como son el pago de las prestaciones por desempleo o cualquier otro tipo de renta asistencial, así como la conveniencia de tener programas que ayuden a los trabajadores desempleados en la búsqueda de un nuevo empleo, como son los dirigidos a la formación o los destinados a fomentar la contratación de determinados grupos de trabajadores más afectados por el desempleo.

Las diferentes reformas laborales que se han puesto en marcha en España desde el año 1997 han tenido muy en cuenta estos efectos negativos asociados a la finalización de una relación laboral y han tratado de buscar la estabilidad a través del fomento de la contratación indefinida. Sin embargo los resultados conseguidos no han sido totalmente satisfactorios pues la temporalidad no se ha reducido en la medida que cabía esperar. La persistencia de la temporalidad y su resistencia a disminuir llevó a la idea de que no bastaba con la reducción de la brecha de costes para aumentar lo suficiente el número de contratos indefinidos. En los resultados obtenidos en los trabajos de Toharia et al. (2005) y Cebrián, Moreno y Toharia (2005) se observaba que la duración de los contratos bonificados era menor que la de los contratos ordinarios, poniendo de manifiesto que no todos los contratos indefinidos gozaban de la misma estabilidad. Entre los resultados se obtenía que la brecha de costes no era el elemento determinante de la segmentación entre temporales e indefinidos. La diferente duración de los distintos tipos de contratos sugería una explicación diferente, en el sentido de que la temporalidad no parecía tener su raíz principalmente en la forma jurídica del contrato, sino que el uso de ese tipo de contrato inestable se destinaba a puestos de trabajo inherentemente inestables.

El hecho de que la población asalariada estuviera formada por un grupo de puestos de trabajo y trabajadores estables, que representaban en torno al 70 por ciento del total, y de un segundo grupo, en torno al 30 por ciento del total, que tenía una situación laboral inestable, recordaba la vieja teoría dualista de los institucionalistas americanos, y principalmente de los trabajos de Michael Piore (Doeringer y Piore, 1971; Piore y Berger, 1980, Piore, 1986). Según esta escuela de pensamiento, existe una "segmentación" del mercado de trabajo entre puestos relativamente más cualificados en los que la estabilidad constituye un elemento sustancial, y puestos relativamente poco cualificados para los que la estabilidad de la relación laboral es indiferente, ya que existen numerosos trabajadores que pueden cubrirlos. Y todo ello, independientemente del tipo de contrato, por lo que algunos contratos indefinidos es posible que se estén utilizando para cubrir puestos de trabajo inestables. 
En la misma línea, Cebrián y Toharia (2008), Cebrián et al. (2011a y 2011b) cuando estudian las trayectorias que acompañan a los contratos indefinidos observan que el contacto con la contratación indefinida no garantiza la estabilidad ni la permanencia en el empleo, al tiempo que ponen de manifiesto la importancia de este tipo de contratación para retener a los trabajadores más productivos. Por otra parte, García Pérez y Rebollo (2009) al evaluar las bonificaciones de fomento del empleo indefinido concluyen que hay un efecto positivo, aunque pequeño, sobre la tasa de transición hacia el empleo estable.

Este artículo se centra en averiguar en qué medida la llegada de la crisis ha afectado al empleo estable que se inició en nuestro país en los años de la expansión económica en los que la tasa de crecimiento de la contratación indefinida fue mayor, 2005 y 2006. El objetivo es detectar si se observan diferencias entre las duraciones de los distintos tipos de contratación indefinida que llevan asociados distintos costes de entrada y salida del empleo, controlando por las causas de baja del contrato.

Los datos que se utilizan en este estudio son el resultado de enlazar la Muestra Continua de Vidas Laborales con datos fiscales (MCVL-F) ${ }^{2}$ desde 2005 hasta 2009, explotada en su versión de panel, con el registro de contratos del Servicio Público de Empleo Estatal (SEPE) ${ }^{3}$.

El artículo se estructura en tres apartados además de esta introducción. El siguiente apartado detalla la base de datos utilizada y el planteamiento general del trabajo. A continuación, en otro apartado se presenta un análisis descriptivo de los diferentes colectivos que se van a analizar para pasar seguidamente a presentar la estimación de los modelos que explican la duración de los contratos indefinidos según sus características y causas de extinción, si ésta tiene lugar. El último apartado presenta las principales conclusiones alcanzadas.

\section{PLANTEAMIENTO Y DATOS PARA EL ANÁLISIS DE LA CONTRATACIÓN INDEFINIDA Y SU ESTABILIDAD}

Cuando se observa la evolución de la contratación temporal e indefinida en España es habitual encontrar que los contratos indefinidos muestran saldos de altas y bajas más bajos que los contratos temporales en las épocas expansivas y

\footnotetext{
${ }^{2}$ Esta versión de la MCVL incorpora los registros obtenidos por la Agencia Tributaria a partir del modelo de tributación 190.

${ }^{3}$ Las autoras agradecen al Servicio Público de Empleo Estatal y a la gerencia de la Seguridad Social su inestimable colaboración para poder disponer de los datos. También a los premios FIPROS por la financiación recibida para la realización del proyecto "Estudio sobre la contratación indefinida y sus costes en el marco de relaciones laborales actual y sus consecuencias para la Seguridad Social" que es el origen del estudio que se presenta aquí.
} 
que dicho saldo caiga durante las crisis de forma muy parecida a la de los temporales. Podría interpretarse que durante la etapa expansiva se contratan menos indefinidos porque son caros de despedir, pero durante las crisis se ajustan por igual indefinidos y temporales. Este hecho se apunta también entre los resultados del trabajo Cebrián et al. (2011a) al observar que el uso que hacen las empresas de los diferentes tipos de contratos indefinidos es tal que la entrada en el empleo estable se ve negativamente afectada por los costes de despido mientras que no ocurre de manera tan clara en la salida.

Los datos del Gráfico 1 muestran la tendencia de la contratación indefinida a partir de los datos de la Encuesta de Coyuntura Laboral sobre altas y bajas en el sector privado no agrario, para el periodo 1998-2011. Se puede apreciar que en 2005, 2006 y en el primer trimestre de 2007 las altas de contratos indefinidos aumentaron hasta alcanzar su máximo nivel, punto a partir del cual las bajas comienzan a superar a las altas que disminuyen, por lo que el saldo neto es negativo ${ }^{4}$.

\section{Gráfico 1}

Altas y bajas de los contratos indefinidos en el sector privado no agrario, valores absolutos en miles (altas iniciales)

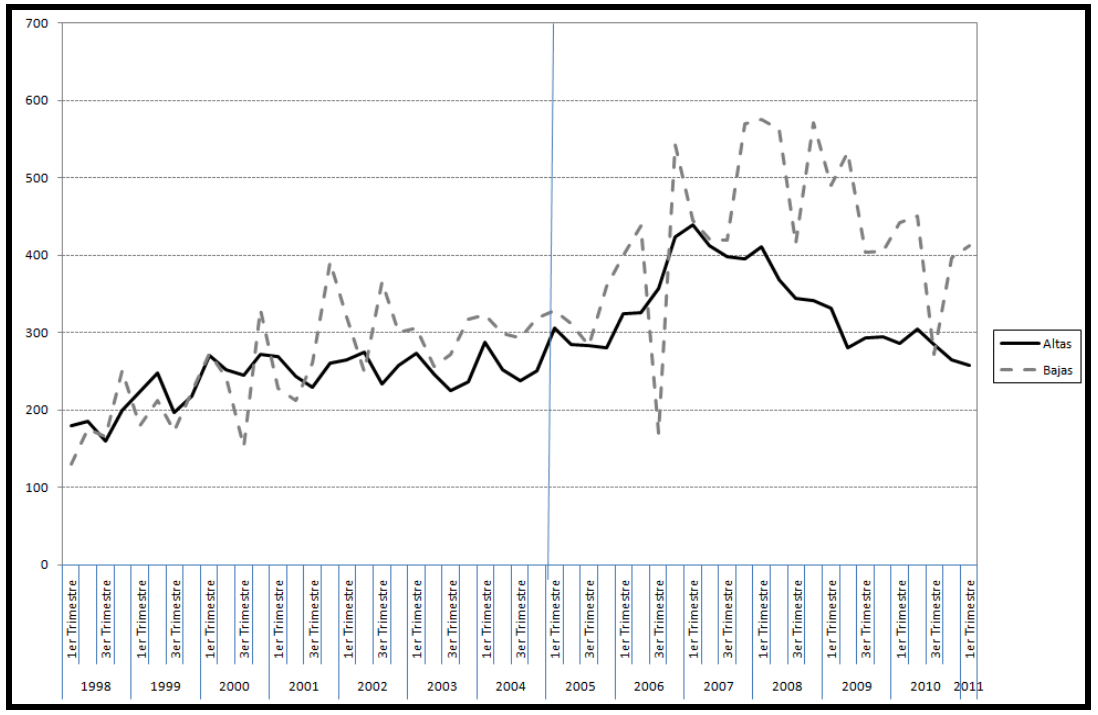

Fuente: Encuesta de coyuntura laboral.

\footnotetext{
${ }^{4} \mathrm{Si}$ se añaden las altas de indefinidos por modificación de contrato el saldo es ligeramente positivo en los años previos a la crisis (Malo y Gonzalez, 2010).
} 
De acuerdo con la información proporcionada por MCVL, el número de episodios de empleo asalariado registrados y vigentes a lo largo de los años del periodo analizado ascendía hasta casi 120 millones. De todos estos episodios, algo menos del 20 por ciento estaba relacionado con un contrato indefinido. Buena muestra de que en el mercado de trabajo español el peso de la contratación temporal es tan importante que a lo largo de las últimas décadas el porcentaje de contratos temporales llegó a superar al 90 por ciento del total de los contratos que se registraban cada año (Toharia, 2011).

La muestra formada por los episodios relacionados con un contrato indefinido es de 945 mil casos, de los cuales casi el 37 por ciento se iniciaron con anterioridad al periodo de referencia, y el 63 por ciento restante comenzó en esos años. En el Cuadro1 se recogen las cifras de distribución de los episodios vigentes en el periodo, en función del momento en el que tuvo lugar el registro de alta o de baja en la Seguridad Social. Se puede apreciar que los contratos que proceden de años anteriores a 2005 continúan dados de alta al finalizar el periodo en un porcentaje mayor (44,5 por ciento) que los iniciados posteriormente, con la única excepción de aquellos que se registraron en 2009 en cuyo caso, la explicación proviene del hecho de que el periodo de observación es menor y de ahí que todavía se mantengan vigentes. Ahora bien, es sorprendente observar cómo más del 28 por ciento de los episodios iniciados cursa baja en el primer año de vida, y cómo este porcentaje va aumentando en los años más recientes hasta tal punto que el 40 por ciento de los iniciados en 2009 habían terminado al finalizar dicho año.

\section{Cuadro 1}

Número de contratos indefinidos registrados en la Seguridad Social que estuvieron vigentes en algún momento del periodo comprendido entre 2005 y 2009 y su distribución según el momento en el que se registró y si cursó baja

\begin{tabular}{|c|c|c|c|c|c|c|c|}
\hline \multirow{3}{*}{ Año de alta } & \multicolumn{6}{|c|}{ Porcentajes fila } & \multirow{3}{*}{$\begin{array}{c}\text { Porcentaje } \\
\text { Columna }\end{array}$} \\
\hline & \multicolumn{5}{|c|}{ Según año de baja } & \multirow{2}{*}{ En alta } & \\
\hline & 2005 & 2006 & 2007 & 2008 & 2009 & & \\
\hline Antes de 2005 & 18,1 & 13,3 & 10,1 & 7,7 & 6,1 & 44,5 & 36,9 \\
\hline 2005 & 28,2 & 21,8 & 12,9 & 8,1 & 5,3 & 23,7 & 12,4 \\
\hline 2006 & & 28,6 & 24,0 & 12,4 & 7,2 & 27,8 & 13,8 \\
\hline 2007 & & & 31,5 & 23,8 & 10,7 & 34,0 & 14,6 \\
\hline 2008 & & & & 36,2 & 21,7 & 42,1 & 12,7 \\
\hline 2009 & & & & & 39,9 & 60,1 & 9,7 \\
\hline Total & 10,2 & 11,6 & 13,2 & 13,6 & 12,1 & 39,3 & 100,0 \\
\hline
\end{tabular}

Fuente: Enlace MCVL-F y registro de contratos, 2005-2009.

Estos datos permiten plantear la posibilidad de que los contratos indefinidos que existen en la actualidad sean más inestables que los que existían antes y que 
su duración no sólo tenga que ver con el efecto que tiene la antigüedad sobre los costes de despido, que explica que los primeros en salir sean los últimos que hayan entrado, sino también por las características de los propios puestos de trabajo y de los trabajadores que están siendo contratados. En efecto, los trabajos de Cebrián et al. (2011a y 2011b) ponen de manifiesto que una parte importante de la contratación indefinida está llegando a puestos de trabajo en los que la rotación parece importar poco, de modo que, en particular, la duración de los contratos indefinidos que se acogen a las medidas de fomento de la contratación indefinida es menor y en especial, si se trata de contratos iniciales bonificados.

Por ello, resulta muy conveniente analizar con detalle el tipo de contrato indefinido y las causas por las que termina. A partir de la información de la MCVL-F es posible identificar si se trata de contratos iniciales o conversiones, y si reciben algún tipo de bonificación a las cuotas de la Seguridad Social, pero no es posible conocer el tipo de indemnización por despido que conllevan. El enlace de la MCVL-F con el registro de contratos resulta muy útil ya que permite incorporar este tipo de información, además del nivel de estudios y la ocupación, que son variables que no están bien recogidas en la MCVL. Ahora bien, este enlace impone una limitación ya que sólo es posible enlazar los episodios de la MCVL con los contratos registrados cada año, por lo que sólo es posible analizar los episodios que se han iniciado cada año. Esto implica dejar fuera del análisis a los contratos indefinidos vigentes en el periodo pero que se iniciaron con anterioridad. No obstante, la muestra resultante permite analizar en qué medida los contratos indefinidos creados en los últimos años del periodo expansivo se han visto afectados por la crisis, así como qué influencia ejercen sobre la duración las características propias de los puestos de trabajo y de los trabajadores.

La tipología de contratos indefinidos utilizada diferencia entre cuatro tipos: contratos iniciales sin bonificación, referidos como contratos ordinarios, iniciales bonificados, conversiones bonificadas y conversiones sin bonificación. Adicionalmente se incorpora la información sobre el tipo de indemnización por despido que tienen, de modo que se clasifican según ésta sea de 45 días por año trabajado con un máximo de 42 mensualidades, referida en ocasiones como "mayor despido", o de 33 días por año trabajado con un máximo de 24 mensualidades, referida como "menor despido".

La realización del enlace de las bases de datos aconseja seleccionar únicamente los contratos indefinidos a tiempo completo, ya que la información sobre ellos es más completa y fiable, así como aplicar algunos filtros que permitan ajustar el tiempo de registro de los contratos. Finalmente la población muestral resultante asciende a 182.258 episodios, con un 45,3 por ciento de casos que continúan vigentes al finalizar el periodo de observación. 
Los motivos que pudieron originar las bajas en el sistema se presentan agregados en las siguientes categorías: baja voluntaria, baja no voluntaria, originada por un despido, y otras causas, grupo en el que se incluyen el resto de los motivos (fusión o absorción, fallecimiento, pensionista, baja de oficio, huelga, excedencias, suspensiones, etc.).

El Cuadro 2 recoge el porcentaje de episodios que cada año cursaron baja según cuál fuese la causa que la originara.

\section{Cuadro 2}

Porcentaje de episodios que se dieron de baja en los años del periodo de referencia según la causa que originó la finalización

\begin{tabular}{|c|c|c|c|c|c|c|c|}
\hline \multirow{2}{*}{$\begin{array}{l}\text { Causa de la } \\
\text { finalización }\end{array}$} & \multirow{2}{*}{$\begin{array}{c}\text { Año de } \\
\text { alta }\end{array}$} & \multicolumn{5}{|c|}{ Año de baja } & \multirow[b]{2}{*}{ Total } \\
\hline & & 2005 & 2006 & 2007 & 2008 & 2009 & \\
\hline \multirow{5}{*}{ Voluntaria } & 2005 & 47,4 & 50,8 & 47,8 & 27,8 & 12,0 & 28,1 \\
\hline & 2006 & & 48,2 & 50,2 & 30,3 & 13,6 & 24,3 \\
\hline & 2007 & & & 47,8 & 35,3 & 17,4 & 20,1 \\
\hline & 2008 & & & & 36,5 & 23,8 & 13,8 \\
\hline & 2009 & & & & & 29,1 & 9,0 \\
\hline \multirow{5}{*}{$\begin{array}{l}\text { Obligatoria } \\
\text { (despido) }\end{array}$} & 2005 & 48,1 & 42,1 & 41,0 & 57,5 & 72,2 & 34,2 \\
\hline & 2006 & & 46,7 & 42,6 & 58,7 & 72,3 & 34,1 \\
\hline & 2007 & & & 48,2 & 57,9 & 71,3 & 34,9 \\
\hline & 2008 & & & & 59,2 & 68,0 & 30,1 \\
\hline & 2009 & & & & & 66,3 & 21,5 \\
\hline \multirow[t]{5}{*}{ Otras } & 2005 & 4,5 & 7,1 & 11,2 & 14,7 & 15,8 & 37,7 \\
\hline & 2006 & & 5,1 & 7,1 & 11,0 & 14,1 & 41,6 \\
\hline & 2007 & & & 4,0 & 6,7 & 11,2 & 45,0 \\
\hline & 2008 & & & & 4,3 & 8,2 & 56,1 \\
\hline & 2009 & & & & & 4,6 & 69,5 \\
\hline
\end{tabular}

Fuente: Enlace MCVL-F y registro de contratos, 2005-2009.

La llegada de la crisis desde finales de 2007 explicaría el aumento del peso de los episodios que registran su baja por un despido, sobre todo en el año 2009. Ahora bien, hay que destacar que más del 40 por ciento de todas las relaciones laborales iniciadas y finalizadas antes de la crisis terminan también como consecuencia de un despido.

Una cuestión interesante que hay que tener en cuenta es que, dado que el periodo total de observación comprende 5 años completos, puede existir alguna relación entre el tiempo disponible de observación desde que tiene lugar el alta y la posible baja, de modo que la censura no afecta por igual a todos los episodios. Esta circunstancia queda anulada cuando se seleccionan los episodios que se registraron entre los años 2005 y 2006 ya que es posible seguirlos a todos du- 
rante los tres años siguientes a su inscripción en el sistema de la Seguridad Social. De esta manera se dispone de una ventana de observación común de 1095 días para todos los episodios que se analizan. Tras realizar esta selección, la muestra final está formada por 55.258 episodios, tal y como aparece en el Cuadro 3 del apartado siguiente.

\section{LA CARACTERIZACIÓN DE LOS EPISODIOS DE EMPLEO INDEFINIDO INICIADOS EN 2005 Y 2006}

A partir de la información de la MCVL-F se puede obtener información sobre la totalidad de episodios de empleo que estuvieron vigentes entre 2005 y 2009. De esta forma es posible conocer en qué situación se encuentran los contratos indefinidos que se firmaron con anterioridad a la crisis.

Antes de proceder a la estimación de los modelos, en el Cuadro 3 se presentan los descriptivos correspondientes a la muestra seleccionada en forma de indicadores de composición o de distribución de los distintos grupos de episodios en función de las variables explicativas que se incorporan en las estimaciones. En las columnas del cuadro se establecen los valores correspondientes al total y a las cuatro salidas posibles definidas según el tipo de situación que se observa al finalizar el periodo. La primera línea del cuadro indica el número de casos muestrales existentes en cada categoría de salida y constituye, pues, la base de los porcentajes que aparecen en el cuadro. También se ha incorporado una primera columna en la que se presentan los valores muestrales correspondientes a cada categoría de las variables explicativas.

La distribución entre hombres y mujeres indica que el porcentaje de mujeres es siempre inferior, aunque se aproxima al 50 por ciento en los casos en los que la salida ha sido por despido o por otras causas.

En cuanto a la edad, son muchos más los trabajadores que tienen 45 años o más y accedieron a un empleo indefinido a través de un episodio que se inició entre 2005 y 2006. En promedio son algo más jóvenes los que finalizan por causas voluntarias o por despido.

En torno a un tercio de los trabajadores que ocupan los puestos de trabajo analizados, tienen estudios secundarios y de ahí, que en todas las posibles salidas sean el grupo predominante. Ahora bien, cabe destacar que cuanto mayor es el nivel de estudios del trabajador, más probable es que se produzca la finalización del episodio por causas voluntarias o por otras causas. Además, en este nivel de estudios predomina el grupo de relaciones laborales que continúan vigentes en el sistema de la Seguridad Social al finalizar el periodo de referencia. 


\section{Cuadro 3}

Descriptivos de los episodios de contratos indefinidos iniciados entre 2005 y 2006 , según situación en el sistema de la Seguridad Social tres años después. Indicadores de composición -porcentajes columna- e indicadores de salida -porcentajes fila-

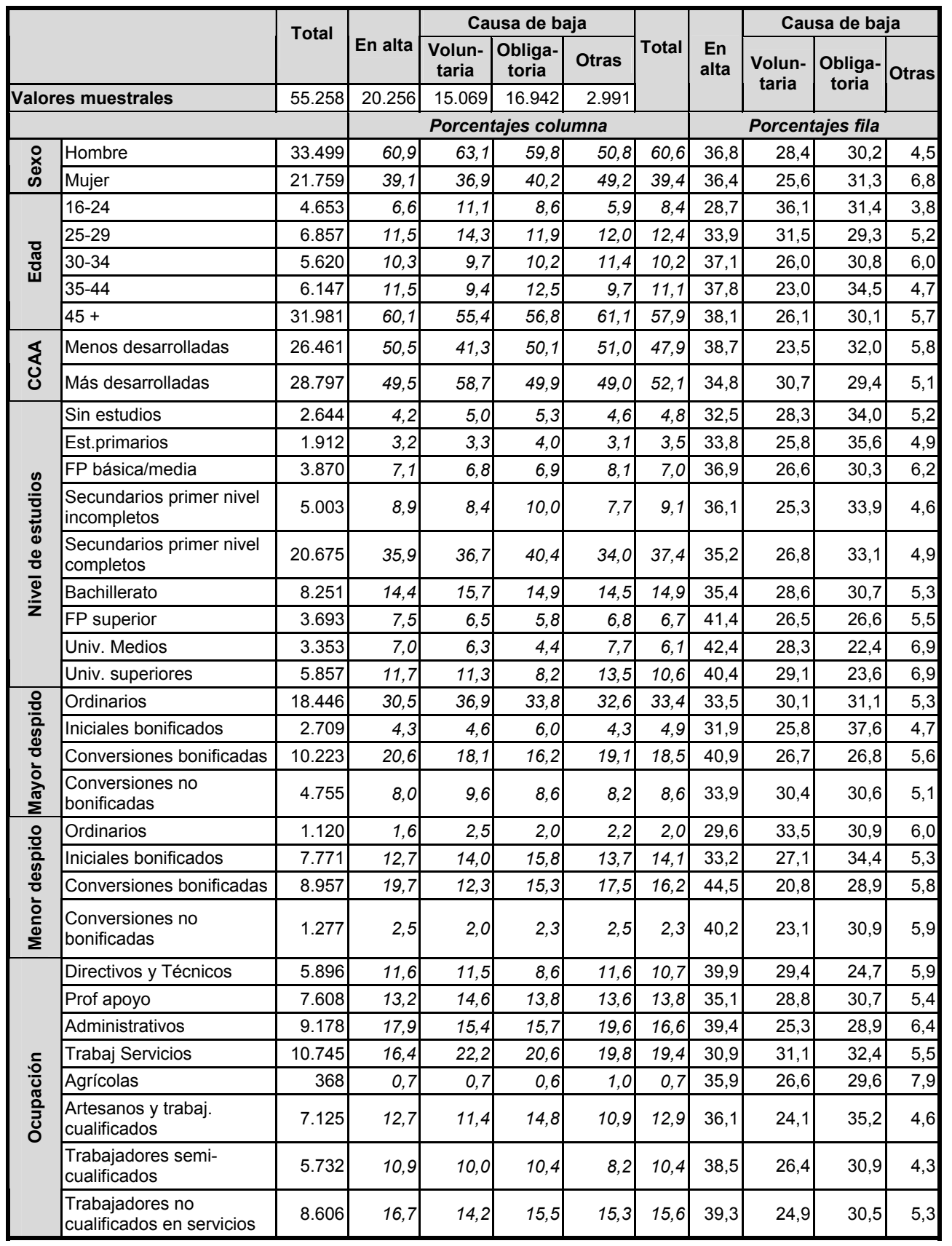




\section{Cuadro 3 (continuación)}

Descriptivos de los episodios de contratos indefinidos iniciados entre 2005 y 2006, según situación en el sistema de la Seguridad Social tres años después. Indicadores de composición -porcentajes columna- e indicadores de salida -porcentajes fila-

\begin{tabular}{|c|c|c|c|c|c|c|c|c|c|c|c|}
\hline & \multirow{2}{*}{ Total } & \multirow[b]{2}{*}{ En alta } & \multicolumn{3}{|c|}{ Causa de baja } & \multirow{3}{*}{ Total } & \multirow{3}{*}{$\begin{array}{l}\text { En } \\
\text { alta }\end{array}$} & \multicolumn{3}{|c|}{ Causa de baja } \\
\hline & & & & $\begin{array}{c}\text { Volun- } \\
\text { taria }\end{array}$ & \begin{tabular}{|c|} 
Obliga- \\
toria
\end{tabular} & Otras & & & Volun- & Obliga- & Otras \\
\hline \multicolumn{2}{|c|}{ Valores muestrales } & 55.258 & 20.256 & 15.069 & 16.942 & 2.991 & & & & & \\
\hline & & & \multicolumn{5}{|c|}{ Porcentajes columna } & \multicolumn{4}{|c|}{ Porcentajes fila } \\
\hline \multirow{9}{*}{ 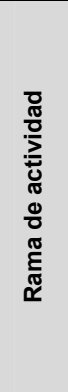 } & Agricultura y Pesca & 488 & 0,8 & 0,9 & 0,8 & 1,5 & 0,9 & 32,4 & 29,3 & 29,3 & 9,0 \\
\hline & Industria y Energía & 8.750 & 19,8 & 11,9 & 14,6 & 15,6 & 15,8 & 45,9 & 20,5 & 28,3 & 5,3 \\
\hline & Construcción & 7.231 & 11,0 & 12,1 & 16,9 & 10,5 & 13,1 & 30,8 & 25,3 & 39,6 & 4,3 \\
\hline & Comercio & 13.051 & 23,9 & 22,8 & 24,0 & 23,5 & 23,6 & 37,1 & 26,4 & 31,1 & 5,4 \\
\hline & Hostelería & 3.929 & 5,0 & 8,6 & 8,7 & 4,6 & 7,1 & 25,8 & 33,2 & 37,5 & 3,5 \\
\hline & Transp. y comunicac. & 6.011 & 10,2 & 13,5 & 9,4 & 10,7 & 10,9 & 34,5 & 33,7 & 26,4 & 5,3 \\
\hline & $\begin{array}{l}\text { Activ Fras,Inmobil. serv. } \\
\text { empresas }\end{array}$ & 10.187 & 17,1 & 21,3 & 17,1 & 20,4 & 18,4 & 34,0 & 31,5 & 28,4 & 6,0 \\
\hline & Servicios colectivos & 3.310 & 8,0 & 4,4 & 4,2 & 10,3 & 6,0 & 49,2 & 19,9 & 21,6 & 9,3 \\
\hline & $\begin{array}{l}\text { Otras actividades y } \\
\text { servicios, hogares }\end{array}$ & 2.301 & 4,1 & 4,4 & 4,3 & 2,9 & 4,2 & 35,9 & 28,7 & 31,6 & 3,8 \\
\hline \multirow{5}{*}{ 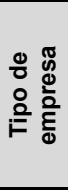 } & Persona física & 6.016 & 7,9 & 11,9 & 13,9 & 9,2 & 10,9 & 26,6 & 29,7 & 39,1 & 4,6 \\
\hline & Sociedad Anónima & 17.114 & 36,4 & 28,4 & 25,4 & 38,7 & 31,0 & 43,1 & 25,0 & 25,1 & 6,8 \\
\hline & Otras sociedades & 29.158 & 47,4 & 56,9 & 57,4 & 42,0 & 52,8 & 32,9 & 29,4 & 33,4 & 4,3 \\
\hline & Cooperativas y similares & 2.044 & 5,3 & 2,3 & 2,5 & 6,7 & 3,7 & 52,4 & 17,2 & 20,6 & 9,8 \\
\hline & Sector público & 926 & 3,0 & 0,5 & 0,8 & 3,3 & 1,7 & 66,0 & 8,1 & 15,1 & 10,8 \\
\hline \multirow{6}{*}{ 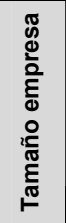 } & 0 & 12.763 & 4,6 & 25,9 & 41,3 & 31,7 & 23,1 & 7,2 & 30,5 & 54,8 & 7,4 \\
\hline & 1 a 9 & 14.807 & 29,0 & 27,0 & 25,9 & 15,8 & 26,8 & 39,7 & 27,4 & 29,7 & 3,2 \\
\hline & 10 a 19 & 5.994 & 12,9 & 11,0 & 8,8 & 7,8 & 10,8 & 43,7 & 27,7 & 24,8 & 3,9 \\
\hline & 20 a 49 & 7.363 & 17,3 & 12,8 & 9,4 & 11,1 & 13,3 & 47,6 & 26,2 & 21,6 & 4,5 \\
\hline & 50 a 249 & 8.072 & 20,2 & 13,0 & 9,0 & 16,1 & 14,6 & 50,8 & 24,3 & 19,0 & 6,0 \\
\hline & 250 o más & 6.259 & 16,0 & 10,4 & 5,5 & 17,6 & 11,3 & 51,7 & 24,9 & 15,0 & 8,4 \\
\hline \multirow{7}{*}{ 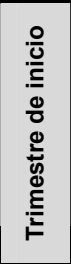 } & 2005 II & 3.012 & 5,0 & 6,2 & 5,4 & 5,2 & 5,5 & 33,4 & 31,1 & 30,3 & 5,2 \\
\hline & 2005 III & 4.773 & 8,5 & 9,1 & 8,4 & 8,6 & 8,6 & 36,1 & 28,6 & 29,9 & 5,4 \\
\hline & 2005 IV & 5.555 & 9,6 & 11,1 & 9,7 & 10,0 & 10,1 & 35,0 & 30,2 & 29,5 & 5,4 \\
\hline & 2006 I & 9.648 & 17,6 & 18,5 & 16,4 & 17,2 & 17,5 & 37,0 & 28,9 & 28,8 & 5,3 \\
\hline & 2006 II & 8.608 & 15,2 & 16,1 & 15,6 & 15,6 & 15,6 & 35,7 & 28,1 & 30,7 & 5,4 \\
\hline & 2006 III & 9.576 & 17,3 & 17,2 & 17,6 & 16,9 & 17,3 & 36,5 & 27,1 & 31,1 & 5,3 \\
\hline & 2006 IV & 14.086 & 26,9 & 21,8 & 27,0 & 26,5 & 25,5 & 38,6 & 23,3 & 32,4 & 5,6 \\
\hline \multirow{5}{*}{ 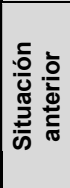 } & Prestación por desempleo & 9.231 & 15,9 & 14,5 & 19,7 & 16,3 & 16,7 & 34,9 & 23,6 & 36,2 & 5,3 \\
\hline & Cuenta propia & 2.079 & 3,8 & 3,6 & 3,9 & 4,0 & 3,8 & 36,6 & 26,1 & 31,6 & 5,7 \\
\hline & Otros empleos & 29.864 & 54,2 & 54,8 & 52,8 & 56,6 & 54,0 & 36,7 & 27,6 & 30,0 & 5,7 \\
\hline & Indefinido & 12.464 & 23,5 & 23,0 & 21,3 & 21,2 & 22,6 & 38,1 & 27,8 & 29,0 & 5,1 \\
\hline & Primer episodio & 1.620 & 2,7 & 4,2 & 2,3 & 1,9 & 2,9 & 33,6 & 38,8 & 24,0 & 3,6 \\
\hline
\end{tabular}

Fuente: Enlace MCVL-F, registro de contratos, 2005-2009.

La información relativa a la comunidad autónoma en la que se encuentra registrada la empresa muestra que hay un número mayor de contratos iniciados en las comunidades más desarrolladas, es decir las que tienen una renta supe- 
rior a la media nacional. Ahora bien, no se distribuyen por igual según el tipo de situación al final del periodo. Así, hay que destacar que el número de bajas voluntarias es mayor en estas comunidades. En cambio, el resto de causas son algo más importantes en las comunidades menos desarrolladas; además, en estas comunidades predomina el grupo de episodios que continúa en alta, seguido del grupo formado por los episodios que han registrado una baja por despido.

En cuanto al tipo de contrato se aprecia que el contrato ordinario predomina por encima de los demás contratos, seguido de las conversiones bonificadas. Y esto es así cualquiera que sea el tipo de causa de salida, aunque si se trata de finalización por despido aumenta el peso de los contratos iniciales y las conversiones. Además, cuando se analiza la distribución se ve que las conversiones son el grupo que mayoritariamente se concentra en los casos que continúan en alta en el sistema.

El tipo de ocupación indica que la composición no es muy diferente atendiendo a la posible salida. Ahora bien, como era de esperar predominan los trabajadores administrativos, de servicios y sin cualificación. En cuanto a la distribución, podría decirse que cuanto menor es el nivel de cualificación, mayor es el peso de los despidos.

La información sobre la rama de actividad en la que se encuadra el episodio analizado indica que la rama de comercio es la más numerosa, y de ahí que tienda a predominar en todos los tipos de salida. Ahora bien, hay que destacar que en ese caso son más numerosas las salidas por despido, y que, por ejemplo, la rama de instituciones financieras e inmobiliarias es más importante en el grupo de episodios con causa de baja voluntaria. En la industria, el comercio y los servicios colectivos predomina que el episodio no haya finalizado; en cambio, en la construcción y en la hostelería predominan las bajas por despido.

Las pequeñas sociedades, las que no son anónimas (colectivas, comanditarias, etc.) son las que mayor peso tienen en todos los grupos, aunque su distribución indica que acogen más casos de baja por despido. En cambio, las sociedades anónimas tienen más episodios que continúan en alta.

En cuanto al tamaño del establecimiento, como era de esperar, los centros que en el momento de la realización de la MCVL no tenían trabajadores son los que predominan en el grupo de salida por despido. Ahora bien, dado que en España existe un predominio de establecimientos de pequeño tamaño, éstos son muy importantes en todas las salidas. Así mismo hay que mencionar que se observa una relación positiva entre el tamaño del establecimiento y el porcentaje de episodios que continúan vigentes.

En lo que se refiere al tipo de situación que tenía con anterioridad el trabajador que ocupa el episodio analizado, llama la atención que la mayoría procedía de otros empleos no indefinidos, y que esta circunstancia es mayoritaria en cualquiera de las salidas, aunque gana algo de peso en las salidas voluntarias. 
Además, provenir de otro empleo indefinido mejora la proporción de este grupo dentro de los episodios que siguen en alta, así como que si se trata de la primera experiencia laboral, aumenta la probabilidad de que se produzca una salida de carácter voluntario.

El momento en el que se inició el episodio dibuja una tendencia creciente en el tiempo al ser mayor el número de episodios inscritos en la Seguridad Social en 2006. En ese año se registran más episodios con finalización por motivos obligatorios por despido y otras causas.

A modo de resumen se puede decir que los descriptivos indican que existen algunas variables claramente relacionadas con que la finalización de un contrato indefinido tenga lugar por una $\mathrm{u}$ otra causa. Muchas de esas variables, aunque no todas ni de forma unívoca, afectan a la probabilidad de que se origine una salida. En el apartado siguiente, se va a tratar de identificar con más detalle el papel que cada una juega en dicha probabilidad de salida y el tiempo que tarda en tener lugar.

\section{ANÁLISIS DE LA PROBABILIDAD DE SALIR DE UN EPISODIO DE EMPLEO INDEFINIDO: RIESGOS EN COMPETENCIA}

Cuando se analiza el tiempo que dura un empleo indefinido, no sólo interesa conocer el papel que juega cada una de las variables explicativas del empleo en su duración, sino también, cuál es la causa que explica que el empleo termine y cómo afectan estas variables a la probabilidad de que se produzca la finalización de la relación laboral por cada uno de esos motivos. Por ello, se va a estudiar el efecto de las características de las personas y de los empleos sobre la duración de la relación laboral y determinar si existen diferencias que expliquen la finalización. Para cada posible tipo de salida, cabe preguntarse qué probabilidad hay de que se produzca cada una de ellas, cómo condicionan la duración del empleo indefinido y qué es lo que puede explicar que se produzcan. Esto significa que de la misma manera que se puede estudiar la duración del empleo, se puede explicar el papel que tiene el motivo que origina que una persona salga de él. Así, a continuación se estudia la duración de un episodio de empleo, diferenciando según la causa que origina su finalización. El tipo de análisis que se realiza es similar al utilizado para estudiar el tiempo que tarda una persona en salir del desempleo teniendo en cuenta cuándo se termina y por qué. El análisis se realiza a partir de la estimación de un modelo que estudia riesgos en competencia (competing risk models, según terminología anglosajona), tal y como se explica a continuación. 


\subsection{Especificación del modelo del análisis de las causas de finalización de un contrato indefinido}

A partir de la información proporcionada por la MCVL-F, se pueden identificar diferentes tiempos de supervivencia dependiendo de cuál sea la razón que explique por qué un episodio que se inició entre el 1 de enero de 2005 y el 31 de diciembre de 2006 continúa vigente tres años después de su inicio. Estos tiempos se determinan en función de cuatro posibles salidas que identifican los sucesos que se suponen en competencia: seguir en alta y estar censurado por la derecha, haber finalizado el episodio por decisión voluntaria del trabajador, haber terminado la relación laboral por causa de despido, o haber tenido cualquier otro motivo para poner fin a la relación. Si además se supone que la correlación entre los factores no observables y que afectan a cada riesgo son independientes es posible estimar modelos de riesgos en competencia.

Los sucesos en competencia se definen de la siguiente manera. La situación de censura (por la derecha) es aquélla que resulta porque en el momento final de observación, es decir, 1095 días después del inicio del episodio con un contrato indefinido a tiempo completo, el episodio se mantiene vigente y, por lo tanto, se encuentra como tal registrado en el sistema de la Seguridad Social. Si no es así, se considera que el episodio ha terminado (fallecido según la terminología de los modelos de duración). Y dado que pueden existir diferentes motivos que expliquen dicha terminación, se utilizan modelos de varias salidas. Los motivos de salida que se definen de acuerdo con las causas que se han detallado son: salida voluntaria, obligatoria por despido o por otras causas. El momento de salida se define como la fecha en la que cursa baja el episodio en el registro de afiliación a la Seguridad Social.

La duración del episodio es una variable continua, ya que se dispone de su valor en días. Se calcula dentro del periodo analizado como la diferencia entre el momento de inicio, es decir, la fecha de alta en el sistema de la Seguridad Social, corregida para tener en cuenta la posible modificación del contrato desde un temporal a un indefinido, y el último momento de observación a lo largo de la ventana definida.

Cuando la variable tiempo es continua, la función de verosimilitud del modelo de varias salidas se convierte en separable (Jenkins, 2005). Así, si las 3 posibles salidas planteadas anteriormente se denominan $\mathrm{V}$ (finalización por causa voluntaria), D (finalización por despido individual) y $\mathrm{O}$ (otros motivos), $\delta^{\mathrm{V}}$, $\delta^{\mathrm{D}}$ y $\delta^{\mathrm{O}}$ son los indicadores binarios de salida (iguales a 1 si se produce la salida e iguales a 0 en caso de censura), $\theta_{\mathrm{V}}, \theta_{\mathrm{D}}$ y $\theta_{\mathrm{O}}$ son las tasas de salida de riesgo de cada uno de los tres motivos posibles y $S_{\mathrm{V}}, S_{\mathrm{D}}$ y $S_{\mathrm{O}}$ son las funciones de supervivencia correspondientes a cada uno de los motivos de salida y $T$ es el tiempo transcurrido hasta la salida (o hasta la censura), el logaritmo de la aportación de 
un episodio de la muestra a la función de verosimilitud puede expresarse de la forma siguiente:

$$
\ln \mathrm{V}=\left\{\delta^{\mathrm{V}} \ln \theta_{\mathrm{V}}(T)+\ln S_{\mathrm{V}}(T)\right\}+\left\{\delta^{\mathrm{D}} \ln \theta_{\mathrm{D}}(T)+\ln S_{\mathrm{D}}(T)\right\}+\left\{\delta^{\mathrm{O}} \ln \theta_{\mathrm{O}}(T)+\ln S_{\mathrm{O}}(T)\right\}
$$

La consecuencia de esta expresión es que la aportación de cada uno de los motivos de la salida a la función de verosimilitud es separable. Por consiguiente, la estimación puede realizarse mediante modelos de destino único para cada uno de los tres destinos. La función de verosimilitud del modelo conjunto de varias salidas será la suma de las funciones de cada uno de los modelos independientes.

Para poder llevar a cabo la estimación, queda por determinar la forma supuesta de las funciones de supervivencia. En el análisis realizado en este trabajo, se ha optado por utilizar dos métodos y comparar sus resultados. Por un lado, se estiman modelos de salida única para cada uno de las tres causas de salida posible, suponiendo que la función de verosimilitud del modelo conjunto de varias salidas será la suma de las funciones de cada uno de los modelos independientes.

En la estimación se han seguido las indicaciones de Jenkins (2005), la forma supuesta de las funciones de supervivencia se ha establecido según el modelo paramétrico de Weibull. El procedimiento planteado suscita el problema de que para que los contrastes de hipótesis relativos a las variables que afectan a las distintas salidas sean válidos, debe cumplirse el supuesto de que las tasas de riesgo deben seguir una forma proporcional. Resulta necesario, pues, realizar los contrastes pertinentes de dicha proporcionalidad. A tal fin, se realizará el contraste propuesto por Narendranathan y Stewart (1991), que se basa en los valores de las funciones de verosimilitud de cada uno de los modelos de salida y el de la función correspondiente al modelo de salida única, junto con el número de salidas observadas a cada uno de los destinos y el número de parámetros estimados en los modelos.

En cuanto a las variables explicativas que se van a utilizar en los modelos son las mismas que se han venido utilizando hasta ahora, aunque en algunos casos ha sido necesario agregar algunas categorías por problemas con el tamaño de las celdas:

- Características personales: sexo, edad, nivel de estudios. La nacionalidad no se ha podido incorporar por no haber casos suficientes de extranjero para que el análisis fuese significativo.

- Características del puesto de trabajo: comunidad autónoma en la que está inscrita la empresa, la rama de actividad, el tipo de empresa, el tamaño de la empresa, el grupo de ocupación y el tipo de contrato indefinido, diferenciando por tipo de coste de despido. 
- Características de las trayectorias: se han incluido dos variables una relativa al tipo de contrato del que procedía el individuo cuando accedió al episodio analizado, y otra que indica cuántos episodios anteriores ha tenido en individuo. También se ha incluido el trimestre de entrada para controlar los posibles factores estacionales y los posibles cambios normativos, así como se considera la tasa de variación del nivel de empleo en dicho trimestre para controlar el ciclo.

\subsection{Modelo de riesgos en competencia según las causas de finalización de un contrato indefinido}

A continuación se presentan los resultados del modelo estimado con el objetivo de incluir en el análisis de la duración de los episodios de empleo indefinido qué ocurre en el momento final de observación, es decir, una vez hayan transcurridos los 1.095 días de la ventana de observación.

Este análisis trata de aportar nuevas evidencias que ayuden a contrastar si la pérdida de un empleo indefinido es un hecho que tiene lugar con cierta frecuencia, tal y como se ha observado en estudios anteriores (Cebrián et al., 2005, Toharia et al. 2005, Cebrián et al, 2011b) y confirmar que los contratos indefinidos tienen un final limitado en un espacio de tiempo relativamente corto.

En el Cuadro 4 se presentan los resultados de los modelos estimados. Entre estas estimaciones se incluye una estimación de la probabilidad conjunta de salida, sin diferenciar por causa de finalización, que sirve para comparar los resultados con los obtenidos en el análisis de la duración del empleo indefinido realizada en otros trabajos (Cebrián et al., 2011b).

En todos los grupos se presentan las tasas de riesgo y el nivel de significatividad de las variables, resultando muchas de ellas significativas. Los valores obtenidos en las tasas de riesgo indican cuando son mayores que uno que es más probable que dicha característica ejerza un efecto positivo sobre la tasa de salida y por tanto, se vea reducida la estabilidad, mientras que si es inferior a uno, indica lo contario, por lo que en ese caso es menor el riesgo de que termine el episodio y por tanto, más probable que la estabilidad se vea favorecida.

Las características de referencia son ser varón, tener entre 16 y 19 años, sin estudios, trabajar en una empresa inscrita en alguna de las comunidades de menor renta, tener un contrato ordinario acogido a mayor despido, ser trabajador no cualificado, en el sector agrícola o pesquero, en una empresa cuyo titular es una persona física, que tiene tamaño muy pequeño (en el momento de la realización de la MCVL-F tenía entre 1 y 9 trabajadores), el contrato se inició en el segundo trimestre de 2005 y el individuo procedía de una situación de desempleo cubierto por una prestación. 


\section{Cuadro 4}

Modelos de duración con varias causas de salida. Modelos Weibull. Episodios de empleo indefinido iniciados en 2005 o 2006

\begin{tabular}{|c|c|c|c|c|c|c|c|c|c|}
\hline & \multicolumn{2}{|c|}{$\begin{array}{l}\text { Modelo salida } \\
\text { (sin causa) }\end{array}$} & \multicolumn{2}{|c|}{$\begin{array}{c}\text { Causa } \\
\text { voluntaria }\end{array}$} & \multicolumn{2}{|c|}{ Despido } & \multicolumn{2}{|c|}{ Otras causas } \\
\hline & & HR & Sig. & HR & Sig. & HR & Sig. & HR & Sig. \\
\hline \multirow[t]{2}{*}{ Sexo } & Hombre (\&) & & & & & & & & \\
\hline & Mujer & 0,960 & ** & 0,789 & *** & 1,044 & * & 1,429 & 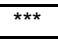 \\
\hline \multirow[t]{5}{*}{ Edad } & $16-19(\&)$ & & & & & & & & \\
\hline & $25-29$ & 0,925 & $\star \star \star *$ & 0,905 & ** & 0,939 & & 1,106 & \\
\hline & $30-34$ & 0,870 & $\star * *$ & 0,778 & $\star \star *$ & 0,938 & & 1,235 & * \\
\hline & $35-44$ & 0,884 & $\star \star \star *$ & 0,814 & $\star \star \star *$ & 0,996 & & 0,935 & \\
\hline & $45+$ & 0,882 & $* * *$ & 0,825 & *** & 0,923 & * & 1,244 & * \\
\hline \multirow[t]{9}{*}{ Nivel de estudios } & Sin estudios (\&) & & & & & & & & \\
\hline & Est.primarios & 0,969 & & 0,983 & & 0,989 & & 0,891 & \\
\hline & FP básica/media & 0,893 & $* * *$ & 0,871 & ** & 0,915 & * & 1,060 & \\
\hline & $\begin{array}{l}\text { Secundarios primer nivel } \\
\text { incompletos }\end{array}$ & 0,918 & ** & 0,906 & * & 0,947 & & 0,838 & \\
\hline & $\begin{array}{l}\text { Secundarios primer nivel } \\
\text { completos }\end{array}$ & 0,934 & ** & 0,916 & * & 0,967 & & 0,914 & \\
\hline & Bachillerato & 0,920 & $\star *$ & 0,913 & * & 0,925 & & 0,934 & \\
\hline & FP superior & 0,822 & $\star \star \star *$ & 0,823 & *** & 0,804 & *** & 0,887 & \\
\hline & Univ. medios & 0,848 & $* * *$ & 0,901 & * & 0,740 & $\star \star \star$ & 1,081 & \\
\hline & Univ. superiores & 0,852 & $* * *$ & 0,857 & $\star \star$ & 0,786 & $\star \star \star *$ & 1,108 & \\
\hline \multirow[t]{2}{*}{$\overline{C C A A}$} & Menos desarrolladas & & & & & & & & \\
\hline & Más desarrolladas & 1,187 & $* * *$ & 1,447 & *** & 1,076 & *** & 0,880 & $* * *$ \\
\hline \multirow[t]{4}{*}{ Mayor despido } & Ordinarios (\&) & & & & & & & & \\
\hline & Iniciales bonificados & 0,972 & & 0,963 & & 1,026 & & 0,765 & ** \\
\hline & Conversiones bonificadas & 0,774 & $\star \star \star *$ & 0,780 & *** & 0,721 & *** & 0,890 & \\
\hline & $\begin{array}{l}\text { Conversiones no } \\
\text { bonificadas }\end{array}$ & 0,913 & *** & 0,935 & * & 0,874 & *** & 0,940 & \\
\hline \multirow[t]{4}{*}{ Menor despido } & ordinarios & 1,041 & & 1,080 & & 0,980 & & 1,215 & \\
\hline & Iniciales bonificados & 0,933 & $\star * *$ & 0,947 & & 0,914 & $\star \star * *$ & 0,899 & \\
\hline & Conversiones bonificadas & 0,689 & $\star \star \star *$ & 0,632 & *** & 0,688 & *** & 0,897 & \\
\hline & $\begin{array}{l}\text { Conversiones no } \\
\text { bonificadas }\end{array}$ & 0,772 & $* * *$ & 0,743 & *** & 0,773 & $* * *$ & 0,965 & \\
\hline \multirow[t]{8}{*}{ Ocupación } & Directivos y Técnicos & 0,938 & ** & 0,952 & & 0,922 & * & 0,907 & \\
\hline & Prof apoyo & 0,995 & & 0,997 & & 0,998 & & 0,941 & \\
\hline & Administrativos & 0,888 & 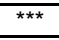 & 0,856 & *** & 0,875 & 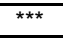 & 0,990 & \\
\hline & Trabaj Servicios & 1,131 & $* * *$ & 1,288 & $\star \star *$ & 1,016 & & 1,116 & \\
\hline & Agrícolas & 1,080 & & 1,059 & & 1,041 & & 1,414 & \\
\hline & $\begin{array}{l}\text { Artesanos y trabajadores } \\
\text { cualificados }\end{array}$ & 0,985 & & 0,995 & & 0,939 & * & 1,026 & \\
\hline & $\begin{array}{l}\text { Trabajadores semi- } \\
\text { cualificados }\end{array}$ & 0,969 & & 1,027 & & 0,970 & & 0,856 & \\
\hline & $\begin{array}{l}\text { Trabajadores no } \\
\text { cualificados en servicios }\end{array}$ & & & & & & & & \\
\hline \multirow{9}{*}{$\begin{array}{l}\text { Rama de } \\
\text { actividad }\end{array}$} & Agricultura y Pesca (\&) & & & & & & & & \\
\hline & Industria y Energía & 0,777 & $* * *$ & 0,562 & $\star * *$ & 1,065 & & 0,548 & *** \\
\hline & Construcc & 0,929 & & 0,712 & $* * *$ & 1,293 & ** & 0,500 & $\star \star * *$ \\
\hline & Comercio & 0,908 & & 0,706 & *** & 1,227 & * & 0,604 & ** \\
\hline & Hostelería & 1,116 & & 0,968 & & 1,487 & $\star \star \star$ & 0,443 & $\star \star \star *$ \\
\hline & Transporte y comunicac. & 1,018 & & 0,958 & & 1,124 & & 0,692 & $*$ \\
\hline & $\begin{array}{l}\text { Actividades fras, inmobil. } \\
\text { serv. empresas }\end{array}$ & 1,047 & & 0,952 & & 1,255 & * & 0,653 & * \\
\hline & Servicios colectivos & 0,860 & * & 0,699 & *** & 0,995 & & 0,731 & \\
\hline & $\begin{array}{l}\text { Otras actividades y } \\
\text { servicios, hogares }\end{array}$ & 0,918 & & 0,807 & * & 1,203 & & 0,422 & *** \\
\hline
\end{tabular}


Cuadro 4 (continuación)

Modelos de duración con varias causas de salida. Modelos Weibull. Episodios de empleo indefinido iniciados en 2005 o 2006

\begin{tabular}{|c|c|c|c|c|c|c|c|c|c|}
\hline & & \multicolumn{2}{|c|}{$\begin{array}{l}\text { Modelo salida } \\
\text { (sin causa) }\end{array}$} & \multicolumn{2}{|c|}{$\begin{array}{l}\text { Causa } \\
\text { voluntaria }\end{array}$} & \multicolumn{2}{|c|}{ Despido } & \multicolumn{2}{|c|}{ Otras causas } \\
\hline & & HR & Sig. & HR & Sig. & HR & Sig. & HR & Sig. \\
\hline \multirow[t]{5}{*}{ Tipo de empresa } & Sociedad Anónima & 0,948 & $\star \star *$ & 0,806 & $\star * *$ & 0,992 & & 1,290 & $* * *$ \\
\hline & Otras sociedades & 1,031 & & 1,009 & & 1,031 & & 0,952 & \\
\hline & Cooperativas y similares & 0,762 & $\star \star *$ & 0,568 & $\star * \star$ & 0,802 & $\star \star \star \star$ & 1,512 & $\star \star \star$ \\
\hline & Sector público & 0,504 & *** & 0,254 & *** & 0,559 & *** & 1,118 & \\
\hline & Individuos (DNIs) (\&) & & & & & & & & \\
\hline \multirow{6}{*}{$\begin{array}{l}\text { Tamaño del } \\
\text { centro }\end{array}$} & 0 (cierre) & 2,293 & $\star * *$ & 1,568 & $\star * *$ & 2,872 & $\star \star \star$ & 3,844 & $\star \star \star *$ \\
\hline & 1 a $9(\&)$ & & & & & & & & \\
\hline & 10 a 19 & 0,935 & $* * *$ & 1,050 & & 0,840 & $* * *$ & 1,138 & \\
\hline & 20 a 99 & 0,859 & $\star * *$ & 1,005 & & 0,724 & $\star * *$ & 1,202 & * \\
\hline & 100 a 249 & 0,792 & $\star \star \star *$ & 0,921 & ** & 0,635 & 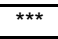 & 1,369 & $\star \star \star *$ \\
\hline & 250 o más & 0,738 & 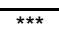 & 0,858 & $\star \star * \star$ & 0,501 & $\overline{* \star \star}$ & 1,794 & $\overline{\star \star \star}$ \\
\hline \multirow{7}{*}{$\begin{array}{l}\text { Trimestre de } \\
\text { inicio }\end{array}$} & 2005 II (\&) & & & & & & & & \\
\hline & 2005 III & 0,951 & & 0,894 & ** & 1,020 & & 1,037 & \\
\hline & 2005 IV & 0,969 & & 0,941 & & 1,048 & & 1,095 & \\
\hline & 2006 I & 0,958 & & 0,926 & & 1,068 & & 1,198 & \\
\hline & 2006 II & 0,970 & & 0,925 & & 1,164 & $\star \star \star *$ & 1,248 & * \\
\hline & 2006 III & 1,013 & & 0,955 & & 1,295 & $\star * *$ & 1,302 & * \\
\hline & 2006 IV & 0,979 & & 0,837 & $\star * *$ & 1,404 & $\star * \star$ & 1,363 & ** \\
\hline \multirow[t]{4}{*}{$\begin{array}{l}\text { Tipo de situación } \\
\text { laboral anterior }\end{array}$} & $\begin{array}{l}\text { Prestación por desempleo } \\
\text { (\&) }\end{array}$ & & & & & & & & \\
\hline & Cuenta propia & 0,873 & $\star * *$ & 0,995 & & 0,752 & $\star * *$ & 1,079 & \\
\hline & Otros empleos & 1,000 & & 1,097 & 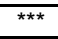 & 0,924 & $\star \star \star$ & 1,067 & \\
\hline & Indefinido & 0,875 & $* * *$ & 0,961 & & 0,807 & 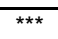 & 0,910 & \\
\hline \multicolumn{2}{|l|}{ Primer episodio } & 0,930 & * & 1,097 & & 0,732 & 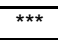 & 0,781 & \\
\hline \multicolumn{2}{|l|}{ Variación empleo } & 1,004 & & 1,001 & & 1,010 & & 0,996 & \\
\hline \multicolumn{2}{|l|}{ Antigüedad } & 0,989 & $\star \star \star *$ & 0,971 & $\star \star \star *$ & 0,997 & $\star \star *$ & 1,008 & $\star \star \star *$ \\
\hline \multicolumn{2}{|l|}{$\mathrm{N}^{\circ}$ epis } & 1,001 & $* * *$ & 1,001 & ** & 1,002 & $* * *$ & 1,000 & \\
\hline
\end{tabular}

(\&) Categoría de referencia. Nivel de significatividad: ***al 99,99 por ciento, ** al 99 por ciento, * al 95 por ciento. Fuente: Enlace MCVL-F, registro de contratos, 2005-2009.

La primera variable incluida es el sexo del individuo que ocupa el empleo al que se refiere el episodio analizado. Se observa que las mujeres tienen una probabilidad mayor de salir por causa de despido u otras causas, mientras que el abandono voluntario, es decir, cuando la ruptura de la relación laboral parte del trabajador, es más probable que sea originada por un hombre. No obstante, recuérdese que la causa voluntaria no incorpora ningún tipo de excedencia, las cuales están en el grupo indeterminado de otras causas y de ahí que sea más probable que la mujer pueda salir por ello.

La edad del individuo es determinante entre las salidas voluntarias, en cuyo caso las estimaciones indican que la edad reduce el riesgo de que se produzca este tipo de rupturas, aunque se observa un cambio de tendencia a partir de los 35 años, de modo que aumenta la probabilidad de que tenga lugar la salida. 
El nivel de estudios se muestra como una variable significativa en las salidas globales, indicando que cuanto mayor sea el nivel de estudios menos probable es que finalice el episodio. Este resultado se obtiene también si se trata de una causa voluntaria; ahora bien, sólo son significativos los niveles más altos de formación si se trata del riesgo de ser despido y no parecen significativos en el resto de las causas.

Si la empresa está inscrita en una comunidad autónoma con un nivel de renta mayor que el correspondiente a la media nacional, aumenta el riesgo de tener una salida de carácter voluntario, aunque también resulta más probable sufrir un despido. En cambio, la probabilidad de salir por otros motivos disminuye.

En relación con los resultados obtenidos sobre el tipo de contrato, en primer lugar, hay que mencionar que resulta interesante poder comparar estos resultados con los estimados en otros trabajos. No obstante, hay que recordar que el análisis que se realiza en esta ocasión se encuentra limitado porque sólo se consideran los episodios que empezaron en los dos primeros años del periodo de observación y se les sigue en un espacio de tiempo de tres años. Por esta razón, no debe sorprender que los resultados que se presentan en los gráficos siguientes reflejen tasas de supervivencia mayores, ya que se trata de contratos que pueden llegar a ser más antiguos al poder verse favorecidos por la etapa expansiva de la economía y a los que la llegada de la crisis tardó más en perjudicarles.

Los gráficos muestran algunas cuestiones interesantes. Por un lado, tanto la salida bruta (Kaplan-Meier), representada en los perfiles del Gráfico 2, como la neta obtenida a partir de la estimación global de la probabilidad de salida sin especificar la causa de la finalización (Gráfico 3), muestran resultados que indican que el tipo de contrato indefinido se muestra muy significativo en todas las estimaciones, especialmente cuando se trata de conversiones de un contrato temporal en uno indefinido. La única excepción es el modelo que analiza la salida por otras causas, ya que sólo son significativamente distintos del contrato ordinario de mayor despido los contratos iniciales bonificados, y que además tienen una tasa de supervivencia mayor ${ }^{5}$.

\footnotetext{
${ }^{5}$ La falta de significatividad de los demás tipos de contrato explica que no se incluya el gráfico correspondiente a esas estimaciones.
} 


\section{Gráfico 2}

Tasas de supervivencia empíricas Kaplan-Meier, ventana de observación de tres años

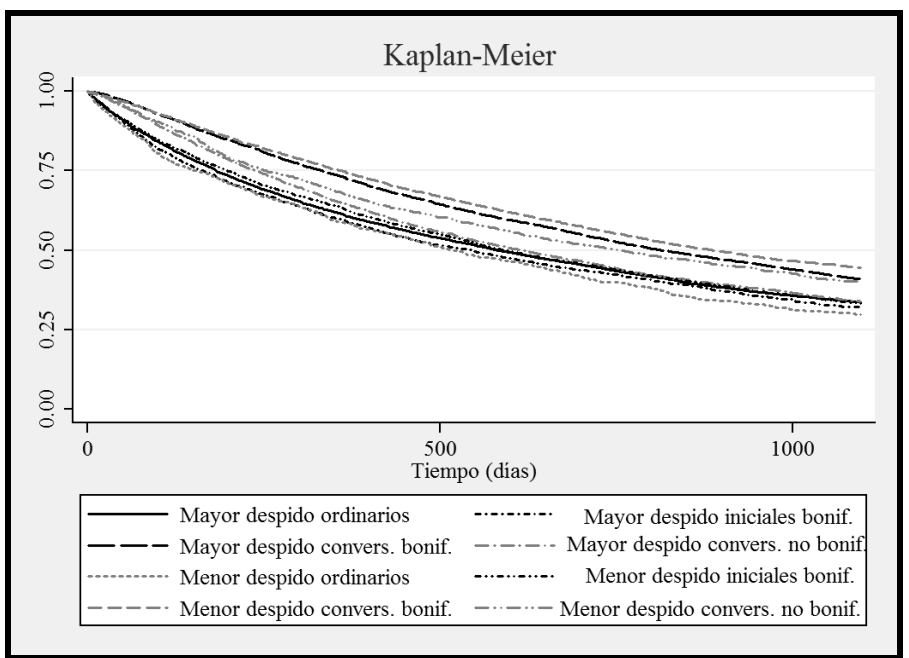

Fuente: Enlace MCVL-F, registro de contratos, 2005-2009.

\section{Gráfico 3}

Tasas de supervivencia estimadas, sin especificar causa de baja, ventana de observación de tres años

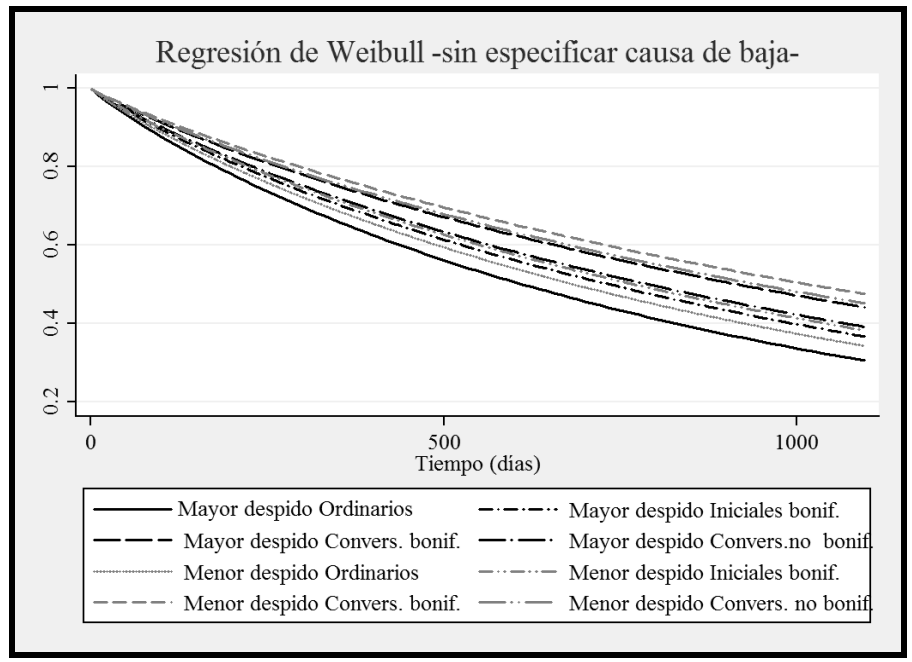

Fuente: Enlace MCVL-F, registro de contratos, 2005-2009.

En todos los demás casos, representados en los Gráficos 4 y 5 , se mantiene el resultado de que los contratos realizados por una conversión son más esta- 
bles. Evidentemente, puede ocurrir que la vigencia de las bonificaciones empuje hacia arriba los resultados dada la ventana de observación, sin embargo, las conversiones no bonificadas mantienen también su posición más favorable, de modo que puede afirmarse una vez más, que los contratos indefinidos por conversión ayudan a alargar la duración de los episodios de empleo indefinido y por lo tanto, su estabilidad ${ }^{6}$. Ahora bien, cuando se consideran los resultados obtenidos en las estimaciones de riesgos en competencia, las tasas de salida cambian, hasta tal punto que mejoran los resultados. En el peor de los casos, las tasas por tipo de contrato dan como resultado que en torno al 60 por ciento de los contratos iniciados entre 2005 y 2006 siguen vigentes a lo largo de la ventana de observación de 1095 días. Ahora bien, se aprecia claramente que cuando la salida tiene lugar por un despido, la probabilidad de mantenerse "vivo" se reduce con respecto a las demás causas de salida. Sí se observa en cambio, que los contratos de fomento del empleo resultan más estables que los contratos ordinarios, resultado sorprendente con respecto a estudios anteriores (Cebrián et al. 2011b), lo que podría venir explicado por el tipo de episodios que se analizan y que pertenecen a los años 2005 y 2006, mientras que en Cebrián et al. (2011b) el análisis se refiere a episodios iniciados entre 2005 y 2009.

\section{Gráfico 4}

Tasas de supervivencia estimadas, causa de baja voluntaria, ventana de observación de tres años

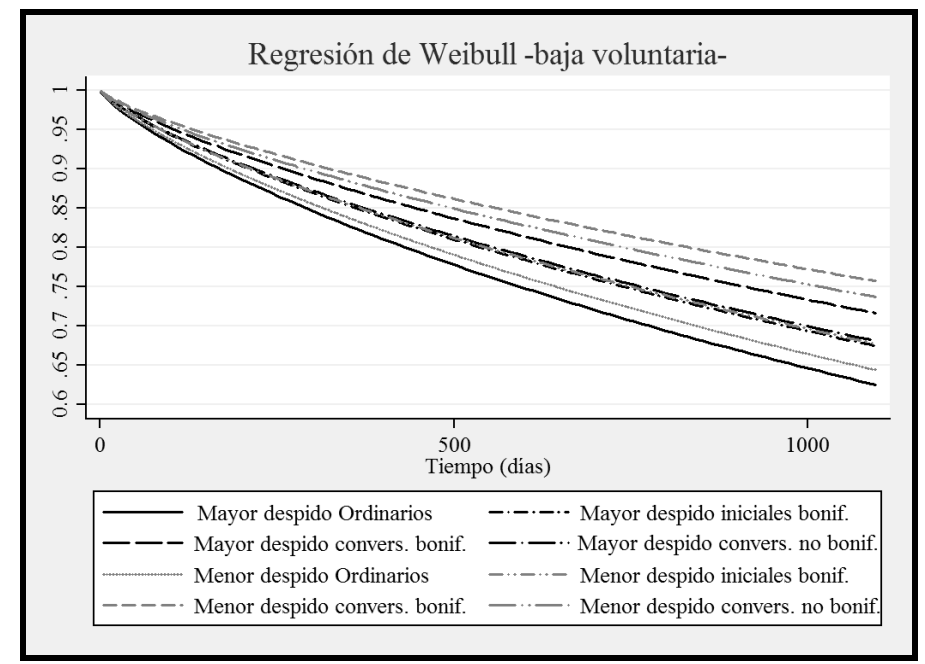

Fuente: Enlace MCVL-F, registro de contratos, 2005-2009.

\footnotetext{
${ }^{6}$ En Cebrián et al. 2011a se suma a la duración del contrato indefinido de conversión la duración del contrato indefinido previo, obteniéndose resultados similares en cuanto a la estabilidad.
} 


\section{Gráfico 5}

Tasas de supervivencia estimadas, causa de baja obligatoria por despido, ventana de observación de tres años

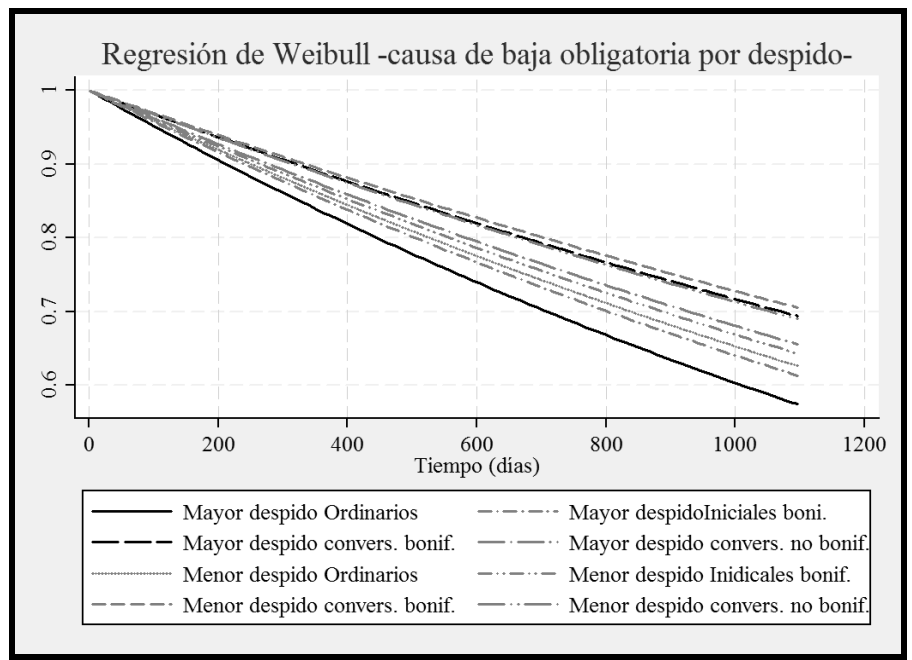

Fuente: Enlace MCVL-F, registro de contratos, 2005-2009.

La ocupación que relaciona la cualificación del individuo con la necesaria para realizar la tarea del puesto de trabajo no parece ser una variable muy significativa. De hecho, sólo los trabajadores que son administrativos parecen tener una probabilidad comparativamente menor que los trabajadores no cualificados de sufrir una ruptura de la relación, ya sea por causa voluntario o por despido.

Los resultados referidos al tipo de actividad económica y sector de la empresa en la que se encuentra el episodio analizado indican que, en términos globales, sólo la industria y energía y los servicios colectivos (sanidad, educación y administración pública) tiene algún tipo de incidencia negativa sobre la probabilidad de que tenga lugar una ruptura de la relación laboral y el episodio deje de estar inscrito. Sin embargo, cuando se concreta por causa de salida, aquellas relaciones que finalizan por algún motivo voluntario, tanto la industria, como la construcción o el comercio, así como los servicios colectivos reducen la probabilidad de que termine el episodio de empleo. En cambio, la construcción, el comercio, la hostelería y las actividades financieras aumentan el riesgo de que la finalización se deba a un despido. Si la razón de la finalización fuesen otras causas, prácticamente en todos los sectores la probabilidad sería menor que en la agricultura.

El tipo de empresa favorece la estabilidad si se trata de sociedades anónimas o cooperativas y mucho si son empresas pertenecientes al sector público (por ejemplo, se reduce a un 25 por ciento la probabilidad de salir por causa volunta- 
ria y al 55 por ciento si se trata de un despido). En cambio, en este tipo de empresas es más probable que se produzcan salidas por otras causas.

En cuanto al tamaño del centro se obtiene lo que cabía esperar, es decir, una relación positiva entre el tamaño y la estabilidad, aunque se rompe si se trata de otras causas. Estos dos tipos de resultados pueden esconder el hecho de que en las sociedades anónimas y en los centros de mayor tamaño es más probable que se lleven a cabo suspensiones de empleo, absorciones y fusiones, por ejemplo.

El trimestre de inicio no es significativo excepto si se trata de despidos, en cuyo caso, haber comenzado el episodio en los tres últimos trimestres de 2006 tiene una probabilidad mayor de que se produzca un despido o finalice la relación por otras causas.

Si el individuo que ocupa el empleo procedía de una prestación es mayor la probabilidad de que finalice el episodio. Ahora bien, si venía de otros empleos no indefinidos, aumenta la probabilidad de que haya una baja voluntaria.

La antigüedad del trabajador en el sistema reduce el riesgo de que termine el episodio por causa voluntaria u obligatoria por despido, aunque aumenta el riesgo de que se produzca una salida por otras causas. En cambio, cuanto mayor sea el número de episodios que haya tenido el trabajador con anterioridad al episodio analizado, más probable es que finalice el contrato. Este resultado puede indicar una vez más que la rotación favorece la rotación.

\section{CONCLUSIONES}

El análisis de la incidencia que tiene sobre la duración de un episodio la probabilidad de que finalice por una $u$ otra causa ha permitido obtener algunos resultados interesantes para explicar la finalización de los contratos indefinidos iniciados en los años 2005 y 2006 al comienzo de la crisis económica.

La contratación indefinida que tuvo lugar en los años 2005 y 2006, sobre todo la generada al amparo de los programas que persiguen la reducción de costes reactivados en la reforma de 2006, muestra un volumen importante de inestabilidad, especialmente debida a despidos, lo que podría explicarse por ir a parar a puestos más inestables, en sectores de baja productividad, como son los sectores de servicios tradicionales como el comercio y la hostelería, así como la construcción, en niveles bajos de ocupación y para trabajadores con poca formación, que evidentemente son los más jóvenes, con menos tiempo en el sistema y sobre todo, varones. Estas evidencias justifican que, a pesar de haber sido años en los que se produjo un aumento importante de contratación indefinida, el stock de indefinidos no aumentara en igual proporción, ya que los contratos duraban poco tiempo, de modo que las tasas relacionadas con la temporalidad prácticamente no se vieron modificadas. 
Estos resultados podrían utilizarse como valoración de lo que puede ocurrir si en la economía española sólo existiese un tipo de relación contractual, cuyas condiciones fuesen variando según fuese ganando antigüedad la relación. Así, por un lado, las empresas tendrían menos inconvenientes en contratar a un trabajador ya que el coste de ponerlo a prueba sería reducido en los primeros meses de la relación, y dado que los costes ligados a la antigüedad serían reducidos, no encontrarían trabas ni para el inicio, ni para la ruptura de la relación. De esta manera, la mayor estabilidad de los contratos indefinidos que son conversiones de un contrato temporal quedaría absorbida por este tipo de contrato único. Ahora bien, de la misma manera, aquellas relaciones más inestables, ya fuese por la falta de ajuste entre el trabajador y el puesto de trabajo, o por las propias características del puesto de trabajo, también se verían absorbidas por este tipo de contrato único, de modo que se favorecería la ruptura de las relaciones contractuales menos justificadas. Estas circunstancias podrían llegar a aumentar tanto el número de contratos iniciados como el número de contratos terminados. Luego, no está garantizado ni el aumento de la estabilidad, ni la reducción de la rotación y, por tanto, tampoco la ruptura de la segmentación.

\section{REFERENCIAS BIBLIOGRÁFICAS}

CEBRIÁN, I.; MORENO, G. y TOHARIA, L. (2005): "Are spanish open-ended contracts permanent? Duration and trajectory analyses", $\mathrm{V}$ Jornadas de Economía Laboral, Alicante.

CEBRIÁN, I. y TOHARIA, L. (2008): La temporalidad en Andalucía: nuevos datos y estudios, Sevilla, Instituto de Estadística de Andalucía.

CEBRIÁN, I. y TOHARIA, L. (2008): "La entrada en el mercado de trabajo español: un análisis basado en la Muestra Continua de Vidas Laborales" en Revista de economía aplicada, $n^{\circ} \mathrm{E}-1$ (vol.XVI): pp. (137-172).

CEBRIÁN, I.; MORENO, G. y TOHARIA, L. (2011a): Evaluación microeconómica de los programas de fomento del empleo indefinido a partir de los datos de los servicios públicos de empleo y de la Muestra Continua de Vidas Laborales, Madrid: Colección Informe y Estudios: Empleo, del Ministerio de Trabajo e Inmigración.

CEBRIÁN, I.; MORENO, G. y TOHARIA, L. (2011b): "La Estabilidad Laboral y los Programas de Fomento de la Contratación Indefinida", Revista de Hacienda Pública, $n^{\circ}$ 198: pp. (103-130)

GARCÍA-PÉREZ, J.I. y REBOLLO, Y. (2009): "The use of permanent contracts across Spanish regions: Do regional wage subsidies work?" en Investigaciones economicas, XXXIII (1): pp. (97-130). 
JENKINS,S.(2005):"Survival Analysis" en ISER, University of Essex, http://www.iser.essex.ac.uk/teaching/degree/stephenj/ec968/pdfs/ec968Inote sv6.pdf

MALO, M.A y GONZALEZ-SANCHEZ, J.J. (2010): "Análisis comparado de los procedimientos de despido: perspectiva económica y jurídica", Ministerio de Trabajo, Madrid.

NARENDRANATHAN, W. y STEWART, M. (1991): "Simple Methods for Testing for the Proportionality of Cause-Specific Hazards in Competing Risk Models" en Oxford Bulletin of Economics and Statistics, vol. 53 (3): pp (331-40).

NICKELL, S. (1997): "Unemployment and labour market rigidities: Europe versus North America" en Journal of Economic Perspectives, 11(3):pp. (5574).

PIORE, M.J. y BERGER, S. (1980): Dualism and discontinuity in industrial societies, Cambridge, Cambridge University Press.

PIORE, M.J. (1986): "Perspectives on labour market flexibility" en Industrial Relations, 2: pp (146-166)

SIEBERT, H. (1997): "Labour market rigidities: at the root of unemployment in Europe" en Journal of Economic Perspectives, 11(3): pp. (37-54).

TOHARIA, L. (en colaboración con Lluís Fina) (1986): "Salarios y costes laborales unitarios en España, 1970-1984", en Enciclopedia de la economía española", n 36 , mayo.

TOHARIA, L. (dir.) (2005): El problema de la temporalidad en España: un diagnóstico, Madrid, Ministerio de Trabajo y Asuntos Sociales.

TOHARIA, L. (2011): "El debate sobre las reformas necesarias para la economía española: el mercado de trabajo" en Gaceta Sindical de CC.OO., 17: pp. (201-236). 
